\title{
Análisis numérico del crecimiento de grieta por fatiga del CPVC: efecto de la temperatura $y$ frecuencia de carga
}

\section{Fatigue crack growth numerical analysis of CPVC: effect of temperature and load frequency}

\author{
Eudi Blanco $^{1}$, Manuel Martínez ${ }^{2}$, Jeanette González ${ }^{3 a}$, Marco González ${ }^{3 b}$ \\ ${ }^{1}$ Universidad Central de Venezuela, Facultad de Ingeniería, Escuela de Ingeniería Metalúrgica y Ciencia de los Materiales. \\ Correo electrónico: eudiblanco@gmail.com \\ ${ }^{2}$ Escuela de Ingeniería Mecánica, Universidad Industrial de Santander, Bucaramanga, Colombia. Orcid: 0000-0001-7069-6400. \\ Correo electrónico: mjmartin@uis.edu.co \\ ${ }^{3}$ Universidad Simón Bolívar, Departamento de Mecánica, Venezuela. Correo electrónico: ajjgonza@ usb.ve, b margdleon@usb.ve
}

Recibido: 22 marzo, 2018. Aceptado: 10 mayo, 2018. Versión final: 21 junio, 2018.

\begin{abstract}
Resumen
En esta investigación se presenta un análisis del efecto combinado de la temperatura y la frecuencia de carga sobre la velocidad de crecimiento de la grieta por fatiga del cloruro de polivinilo clorado (CPVC). El CPVC es un material termoplástico utilizado para sistemas de tuberías en los que la temperatura y la resistencia química son importantes, características que lo convierten en una buena alternativa frente a los metales. El método dual de elementos de contorno (MDEC) se utiliza para determinar estados tridimensionales de esfuerzos y deformaciones en cada incremento de grieta. Los factores de intensidad de esfuerzos en la punta de la grieta se determinan usando la integral J. Un modelo matemático propuesto por Kim \& Wang en 1994, basado en resultados experimentales, se aplica para predecir la velocidad de crecimiento. Para ello, se evalúa una muestra con una grieta lateral utilizando valores de temperatura entre 23 y $70{ }^{\circ} \mathrm{C}$ y frecuencias entre 0,1 y $10 \mathrm{~Hz}$. Los resultados muestran que la velocidad de crecimiento aumenta con el aumento de la temperatura y con la disminución de la frecuencia. Los resultados se comparan con el modelo de París y Erdogan y se revela una relación entre ellos, lo que implica que el MDEC podría ser una herramienta adecuada y precisa para investigar la predicción del crecimiento de la grieta en los polímeros.
\end{abstract}

Palabras clave: CPVC; método de los elementos de contorno; mecánica de la fractura; propagación de grietas por fatiga; temperatura y frecuencia de carga.

\begin{abstract}
An analysis of combined effect of temperature and load frequency on the fatigue crack growth rate and cracking velocity for Chlorinated Polyvinyl Chloride (CPVC) is presented in this paper. CPVC is a thermoplastic material used for piping systems where higher temperature and chemical resistance are important, becoming a good alternative to metals. The Dual Boundary Element Method (DBEM) is used to determine three dimensional states of stresses and strains at each increment of crack. Stress intensity factors at the crack tip are determined using the J-integral, and the crack growth direction is defined by the maximum principal stress criteria. A mathematical model proposed by Kim \& Wang in 1994 based on experimental results is applied to predict cracking velocity. In this work, a specimen with lateral crack using temperature values between 23 and $70{ }^{\circ} \mathrm{C}$ and frequencies between 0.1 and $10 \mathrm{~Hz}$ is evaluated. The results show that cracking velocity increases with temperature increase and with frequency decrease. These results are compared to Paris \& Erdogan model showing good agreement which reveals that DBEM could be an accurate tool to investigate the prediction of crack growth in polymers.
\end{abstract}

ISSN impreso: 1657 - 4583, ISSN en línea: 2145 - 8456, CC BY-ND 4.0 (c) (1) ()

Como citar: E. Blanco, M. Martínez, J. González, M. González, "Análisis numérico del crecimiento de grieta por fatiga del CPVC: efecto de la temperatura y frecuencia de carga," Rev. UIS Ing., vol. 18, no. 1, pp. 177-186, 2019. doi: 10.18273/revuin.v18n1-2019016 
Keywords: CPVC; fracture mechanic; fatigue crack growth; load frequency and temperature; the boundary element method.

\section{Introducción}

El fenómeno de fatiga se puede definir como la disminución de la resistencia mecánica de los materiales al someterlos a esfuerzos cíclicos que favorecen el crecimiento estable de una grieta. El comportamiento de fatiga de muchos polímeros es sensible a las condiciones de ensayo, tales como la frecuencia de aplicación de la carga y la temperatura [1].

El material polimérico empleado para este estudio fue el policloruro de vinilo clorado (CPVC), el cual, por su bajo índice de cristalinidad, puede considerarse un polímero altamente amorfo, que lo hace merecedor de características isotrópicas. Por otra parte, su alta resistencia térmica le otorga al CPVC un buen desempeño en aplicaciones que demandan altas temperaturas, razón por la cual permite considerar este polímero como termoelástico.

El efecto de la temperatura sobre la velocidad de crecimiento de grieta por fatiga (VCG) de diversos polímeros ha sido objeto de estudio de algunos trabajos, de los cuales se ha obtenido que un incremento de la temperatura está asociado con un aumento de la velocidad de propagación de grieta.

Kim y Mai [2] estudiaron el efecto de la temperatura sobre la resistencia al crecimiento de grieta del policloruro de vinilo no plastificado (uPVC) en un rango de temperatura entre -30 y $50{ }^{\circ} \mathrm{C}$. Ellos obtuvieron que la resistencia al crecimiento de grieta disminuye con el incremento de la temperatura y que la fractura frágil es dominante a altas temperaturas, mientras que la fluencia al corte es dominante a bajas temperaturas. Resultados similares obtuvieron Irfan-ul-Haq y Merah [3] para el $\mathrm{CPVC}$ a temperaturas de ensayo entre -10 y $70{ }^{\circ} \mathrm{C}$.

Con respecto al efecto de la variación de la frecuencia de carga sobre la VCG, Hertzberg et al. [4] mostraron que, a temperatura ambiente, un aumento en la frecuencia está asociado con una disminución de la velocidad de propagación de grieta en el PVC. Estos investigadores también reportaron que el poliestireno (PS) y la polisulfona (PSF) son sensibles a la frecuencia de carga a bajas temperaturas de ensayo, mientras que el polimetilmetacrilato (PMMA) es menos sensible a la frecuencia de carga a bajas temperaturas.

Mientras muchos polímeros son sensibles a la frecuencia de ensayo a temperatura ambiente, otros, como el policarbonato (PC), el policloruro de vinilo clorado
(CPVC) y la polisulfona (PSF), no muestran sensibilidad de la velocidad de propagación de grieta con la frecuencia de ensayo a temperatura ambiente.

Kim y Wang [5] estudiaron el efecto combinado de la temperatura y la frecuencia sobre el crecimiento de grieta en el uPVC. Ellos propusieron un modelo matemático, basado en la ecuación de Arrhenius y la energía de activación, para predecir la velocidad de propagación de grieta $d a / d N$ como una función de la temperatura y la frecuencia, donde $d a$ es la variación de la longitud de grieta, cuya longitud inicial es $a_{0}$, a medida que ocurre la variación del número de ciclos $d N$.

Parson [6] estudió el efecto de la temperatura y la frecuencia sobre la velocidad de propagación de grieta del PVC y, además de obtener que un aumento de la temperatura de ensayo y una disminución de la frecuencia causan, por separado, un incremento de la velocidad de crecimiento de grieta, llegó a la conclusión de que, a bajas frecuencias $(0,01 \mathrm{~Hz})$ y altas temperaturas, el crecimiento de grieta por creep es el mecanismo dominante. Similares resultados se obtuvieron para el polietileno de alta densidad (PEAD).

Saghir et al. [7] modelaron el efecto combinado de la temperatura y la frecuencia sobre el crecimiento de grieta en la fatiga del CPVC, empleando el modelo matemático propuesto por Kim y Wang [5], basado en la ecuación de Arrhenius, y obtuvieron una buena predicción de las velocidades de crecimiento de grieta en el CPVC a varias temperaturas y frecuencias.

El uso del análisis computacional para el modelado de la mecánica de fractura ha sido aceptado como un buen reemplazo para ensayos de laboratorios y pruebas piloto, con el objetivo de disminuir los costos asociados al diseño de nuevos elementos y el mantenimiento de elementos existentes [8]. Específicamente, según Mackerle [9], el área de mecánica de fractura permitirá desarrollar un importante número de códigos de computación de propósitos especiales basados en el método de elementos de contorno (MEC). Todo este desarrollo se inicia cuando Cruse presenta artículos de análisis de esfuerzos en sólidos con grietas basados en la resolución numérica de las ecuaciones integrales de contorno relacionadas con problemas de elastostática tridimensional [10, 11]. El MEC, en conjunto con el método de diferencias finitas, fueron los primeros métodos numéricos en ser aplicados a problemas de mecánica de fractura, mientras que se conoce que el método de los elementos finitos fue aplicado a fractura por primera vez en 1971 [12]. 
Estos primeros trabajos de Cruse reportaron poca precisión en los resultados para la obtención de factores de intensidad de esfuerzos (SIF). En general, las primeras aplicaciones del MEC a problemas de grietas estuvieron limitadas por el hecho de que las dos superficies que conforman la grieta en un sólido son coplanares, lo que genera una degeneración matemática [12].

Luego del esfuerzo de muchos investigadores, a principios de la década de los noventa, Portela et al. [13] y Mi y Aliabadi [14], en cuanto a cuerpos 2D y sólidos $3 \mathrm{D}$, respectivamente, publicaron las bases del método dual de los elementos de contorno (MDEC), que implica la aplicación de la ecuación integral de contorno formulada en términos de desplazamientos en una superficie de la grieta y la ecuación integral de contorno formulada en términos de tensiones en la otra superficie de la grieta, para evitar de esta manera la degeneración matemática intrínseca en la formulación de Kelvin.

El método dual de los elementos de contorno (MDEC) ha demostrado ser una alternativa eficaz en el estudio y la simulación de crecimiento de grietas, tanto en la precisión de sus resultados como en la disminución de tiempo de preproceso y construcción del modelo, lo que lo hace sobresalir por encima de otros métodos computacionales de análisis, como el método de los elementos finitos o el método de diferencias finitas [8].

En este trabajo se presenta la simulación computacional del crecimiento de grieta por fatiga del CPVC, empleando el modelo matemático propuesto por Kim y Wang [4], para evaluar el efecto de la temperatura y la frecuencia de carga sobre la velocidad de propagación de la grieta y el MDEC en tres dimensiones para termoelasticidad como herramienta numérica para el cálculo de los parámetros fractomecánicos necesarios para el análisis. Se desarrolló con este propósito un algoritmo computacional en lenguaje Fortran ${ }^{\circledR}$, que contiene un programa basado en la formulación del MDEC. La versatilidad y la eficiencia de la metodología propuesta se demuestra mediante un caso que comprende una geometría con frente de grieta recto.

\section{Método dual de los elementos de contorno}

En el análisis de mecánica de fractura computacional, una grieta se modela como dos superficies coplanares; esto hace que la aplicación del método de los elementos de contorno en las superficies de la grieta, bajo modo mixto de cargas, produzca una degeneración numérica de la formulación (matriz singular), por la aplicación de un mismo tipo de ecuación integral a dos superficies coincidentes, ya que los puntos de colocación sobre las caras son idénticos $[8,12]$.
El MDEC sobrelleva el problema de modelado al incorporar dos ecuaciones independientes, la ecuación integral de desplazamientos y la ecuación integral de tracciones sobre cada una de las caras de la grieta. De esta forma, y aunque los puntos de colocación sobre las caras de la grieta continúan siendo coincidentes, sus respectivas ecuaciones integrales son diferentes.

En la figura 1 se presenta un dominio lineal elástico, isotrópico y homogéneo limitado por su contorno $\Gamma(x)$.

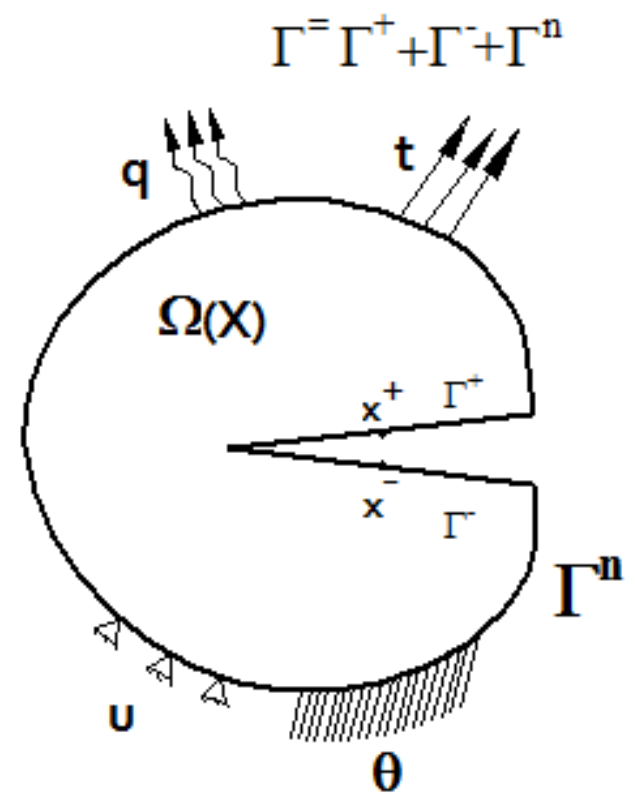

Figura 1. Modelo de fractura con condiciones de contorno. Fuente: elaboración propia.

Las ecuaciones que gobiernan la termoelasticidad en estado estacionario son la ecuación de conducción de Laplace (1) y la ecuación de Navier (2).

$$
\begin{gathered}
\theta_{, k k}=0 \\
\mu u_{i, j j}+\frac{\mu}{1-2 v} u_{j, j i}+b_{i}=\frac{2 \mu v(1+v)}{(1-2 v)} \alpha \theta_{, i}
\end{gathered}
$$

donde $\theta$ es la temperatura, $u_{i}$ los componentes de desplazamiento, $\mu$ es el módulo de corte, $v$ es la relación de Poisson y $\alpha$ es el coeficiente lineal de expansión térmica.

En el caso más general, las ecuaciones diferenciales (1) y (2) están sujetas a condiciones de contorno de temperatura en $\Gamma_{\theta}$ y flujo de calor $q$ en $\Gamma_{q}$ y a condiciones de desplazamiento en $\Gamma_{u} \mathrm{y}$ tracción $\Gamma_{t}$ en $t$. 
La aplicación del MDEC a problemas de fractura termoelásticos requiere las ecuaciones integrales de contorno, las ecuaciones de Laplace (1) y de Navier (2). De acuerdo con el trabajo de Dell'Erba [15], las ecuaciones integrales que relacionan la temperatura $\theta(x)$ y flujo de calor $q(x)$ en el contorno $\Gamma(x)$ son

$$
\begin{gathered}
c\left(x^{\prime}\right) \theta\left(x^{\prime}\right)-\int_{\Gamma} q^{*}\left(x^{\prime}, x\right) \theta(x) d \Gamma=-\int_{\Gamma} \theta^{*}\left(x^{\prime}, x\right) q(x) d \Gamma \\
c\left(x^{\prime}\right) q\left(x^{\prime}\right)+n_{i}\left(x^{\prime}\right) \int_{\Gamma}^{C P V} \theta_{i}^{* *}\left(x^{\prime}, x\right) q(x) d \Gamma=n_{i}\left(x^{\prime}\right) \int_{\Gamma}^{H P V} q_{i}^{* *}\left(x^{\prime}, x\right) \theta(x) d \Gamma
\end{gathered}
$$

donde $\theta^{*}\left(x^{\prime}, x\right), q^{*}\left(x^{\prime}, x\right), \theta_{i}^{* *}\left(x^{\prime}, x\right)$ y $q_{i}^{* *}\left(x^{\prime}, x\right)$ denotan las soluciones fundamentales de las temperaturas y flujos de calor en el punto $x$ debidas a una fuente de calor puntual colocada en la posición $x^{\prime}$. El término $c\left(x^{\prime}\right)$ es una constante que depende de la geometría local, que para un contorno suave $c=1 / 2$. En el caso de la ecuación de flujo $n_{i}\left(x^{\prime}\right)$, corresponde al vector normal al contorno en el punto de colocación, se supone que en las superficies de la grieta $n_{i}\left(x^{+}\right)=-n_{i}\left(x^{-}\right)$.

La ecuación integral de desplazamiento y la de tracción se escriben como

$$
\begin{aligned}
& c_{i j}\left(x^{\prime}\right) u_{j}\left(x^{\prime}\right)+\int_{\Gamma}^{C P V} T_{i j}\left(x^{\prime}, x\right) u_{j}(x) d \Gamma-\int_{\Gamma} \bar{P}_{i}\left(x^{\prime}, x\right) \theta(x) d \Gamma \\
& =\int_{\Gamma} U_{i j}\left(x^{\prime}, x\right) t_{j}(x) d \Gamma-\int_{\Gamma} \bar{Q}_{i}\left(x^{\prime}, x\right) q(x) d \Gamma
\end{aligned}
$$$$
\frac{1}{2} t_{i}\left(x^{\prime}\right)+n_{j}\left(x^{\prime}\right) \int_{\Gamma}^{H P V} T_{k j}\left(x^{\prime}, x\right) u_{k}(x) d \Gamma-n_{j}\left(x^{\prime}\right) \int_{\Gamma}^{C P V} \bar{P}_{i j}\left(x^{\prime}, x\right) \theta(x) d \Gamma+\frac{\mu(1+v)}{(1-2 v)} \alpha n_{i}\left(x^{\prime}\right) \theta\left(x^{\prime}\right)
$$$$
=n_{j}\left(x^{\prime}\right) \int_{\Gamma}^{c P V} U_{k j}\left(x^{\prime}, x\right) t_{k}(x) d \Gamma-n_{j}\left(x^{\prime}\right) \int_{\Gamma} \bar{Q}_{i j}\left(x^{\prime}, x\right) q(x) d \Gamma
$$

donde $u_{j}$ y $t_{i}$ representan los valores de desplazamiento y tracción en el contorno, respectivamente. $U_{i j}\left(x^{\prime}, x\right)$ y $T_{i j}\left(x^{\prime}, x\right)^{\prime}$ son las soluciones fundamentales de Kelvin y $P_{i}\left(x^{\prime}, x\right)$ y $Q_{i}\left(x^{\prime}, x\right)$ son las soluciones fundamentales obtenidas al reducir las fuerzas de origen térmico del volumen al contorno.

Estas ecuaciones son aplicadas al sólido de la siguiente manera:

- Las ecuaciones integrales de desplazamiento (5) y de temperatura (3) se aplican para los puntos de colocación en una superficie de la grieta.

- Las ecuaciones integrales de tracción (6) y de flujo (4) se aplican para los puntos de colocación en la superficie opuesta de la grieta.

- Las ecuaciones integrales de desplazamiento (5) y

de temperatura (3) se aplican para los puntos de colocación en el resto de la superficie del modelo.
En la figura (2) se presenta el esquema general de la estrategia de discretización empleada para el mallado de la geometría.

La metodología usual para resolver problemas de fractura consiste en tratar por separado los sistemas de ecuaciones resultantes de la @̧ưuación de potencial y elasticidad, sin embargo, ambos sistemas pueden acoplarse, según lo hecho por Balderrama [16].

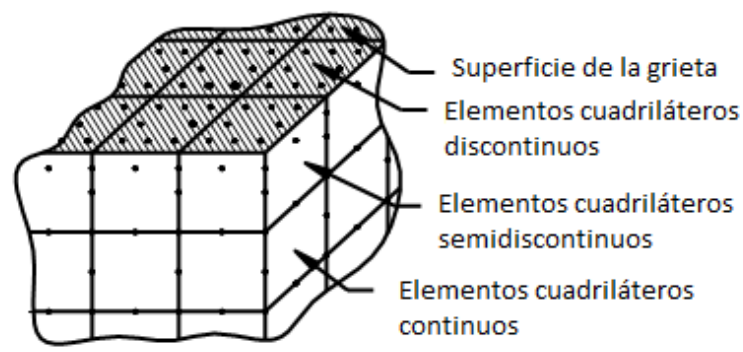

Figura 2. Estrategia de discretización. Fuente: elaboración propia.

La ecuación matricial resultante de la discretización de las ecuaciones es

$$
\left\lfloor H^{\alpha}\right\rfloor\left\{u_{\alpha}\right\}=\left\lfloor G^{\alpha}\right\rfloor\left\{\mathrm{t}_{\alpha}\right\}
$$

donde:

$$
\begin{gathered}
\left\{u_{\alpha}\right\}=\left\{\begin{array}{l}
u_{1} \\
u_{2} \\
u_{3} \\
\theta
\end{array}\right\} \quad\left\{t_{\alpha}\right\}=\left\{\begin{array}{l}
t_{1} \\
t_{2} \\
t_{3} \\
q
\end{array}\right\} \\
{\left[H^{\alpha}\right]=\left[\begin{array}{llll}
H_{11} & H_{12} & H_{13} & H_{1 \theta} \\
H_{21} & H_{22} & H_{23} & H_{2 \theta} \\
H_{31} & H_{32} & H_{33} & H_{3 \theta} \\
0 & 0 & 0 & H_{\theta \theta}
\end{array}\right]} \\
{\left[G^{\alpha}\right]=\left[\begin{array}{cccc}
G_{11} & G_{12} & G_{13} & G_{1 \theta} \\
G_{21} & G_{22} & G_{23} & G_{2 \theta} \\
G_{31} & G_{32} & G_{33} & G_{3 \theta} \\
0 & 0 & 0 & G_{\theta \theta}
\end{array}\right]}
\end{gathered}
$$

A través de las condiciones de contorno se realiza un intercambio entre las columnas de las matrices de la ecuación (7) y se produce un sistema de la forma siguiente:

$$
[A]\{x\}=\{b\}
$$

donde la matriz $A$ engloba algunos coeficientes de las matrices $H$ y $G, x$ es un vector con las incógnitas del 
problema (desplazamientos, tracciones, temperaturas y flujos, según se hayan especificado las condiciones de contorno) y $b$ un vector que resulta del producto de los coeficientes de las matrices $H$ y $G$ por los valores conocidos de los vectores $u$ y $t$ de las condiciones de contorno.

Una vez conocidas las variables de temperatura/flujo de calor y desplazamiento/tracción en el contorno del dominio, se deben realizar cálculos de posproceso para determinar las variables requeridas en el cálculo de la integral $\mathbf{J}$ de dominio: derivadas de desplazamiento y temperatura, esfuerzos y deformaciones $\left(u_{i, j}, \theta, k, \sigma_{i j}\right.$ y $\left.\varepsilon_{i j}\right)$.

El método de elementos de contorno resulta muy versátil en este aspecto, ya que permite calcular las variables a partir de sus correspondientes representaciones integrales. De esta forma, en un punto $x$ ' perteneciente al dominio del problema, la derivada de la temperatura se determina derivando la ecuación (3) [15]:

$\theta_{, k}\left(x^{\prime}\right)-\int_{\Gamma} q_{, k}^{*}\left(x^{\prime}, x\right) \theta(x) d \Gamma=-\int_{\Gamma} \theta_{, k}^{*}\left(x^{\prime}, x\right) q(x) d \Gamma(9)$

Las derivadas de los desplazamientos en puntos internos son calculadas a partir de la ecuación integral de contorno, obtenida al derivar analíticamente la ecuación integral de desplazamiento (5):

$$
\begin{aligned}
& u_{i, k}\left(x^{\prime}\right)+\int_{\Gamma} T_{i j, k}\left(x^{\prime}, x\right) u_{j}(x) d \Gamma-\int_{\Gamma} \bar{P}_{i, k}\left(x^{\prime}, x\right) \theta(x) d \\
& =\int_{\Gamma} U_{i j, k}\left(x^{\prime}, x\right) t_{j}(x) d \Gamma-\int_{\Gamma} \overline{Q_{i, k}}\left(x^{\prime}, x\right) q(x) d \Gamma
\end{aligned}
$$

donde

$T_{i j, k}\left(x^{\prime}, x\right), U_{i j, k}\left(x^{\prime}, x\right), \bar{P}_{i, k}\left(x^{\prime}, x\right)$ y $\bar{Q}_{i, k}\left(x^{\prime}, x\right)$ son las derivadas de los campos fundamentales.

Finalmente, las deformaciones y los esfuerzos se pueden calcular utilizando la definición del tensor de deformaciones infinitesimales y la ley de Hooke generalizada para problemas termoelásticos:

$$
\varepsilon_{i j}=\frac{1+v}{E} \sigma_{i j}-\frac{v}{E} \sigma_{k k} \delta_{i j}+\alpha \theta \delta_{i j}
$$

donde

$$
\sigma_{i j}=\frac{E}{1+v}\left(\varepsilon_{i j}+\frac{v}{1-2 v} \varepsilon_{k k} \delta_{i j}\right)-\frac{E}{1+v} \alpha \theta \delta_{i j}
$$

\section{Cálculo de los factores de intensidad de esfuerzos}

Mediante los resultados obtenidos por el MDEC, los factores de intensidad de esfuerzo $K_{I}, K_{I I} y K_{I I I}$ se determinaron numéricamente aplicando la integral $\mathbf{J}$ de dominio para termoelasticidad sobre un volumen definido en el frente de grieta [16] (figura 3).

La integral $J$ está definida por la siguiente expresión:

$$
\bar{J}=\int_{V}\left\{\left(\sigma_{i j} u_{j, k}-W \delta_{k i}\right) q_{k, i}+\sigma_{n n} \alpha \theta_{, k} q_{k}\right\} d V
$$

Donde $W$ es la densidad de energía de deformación, $q_{k}$ es una función auxiliar y $q_{k, i}$ es la derivada parcial de $q$ con respecto a la coordenada $i$.

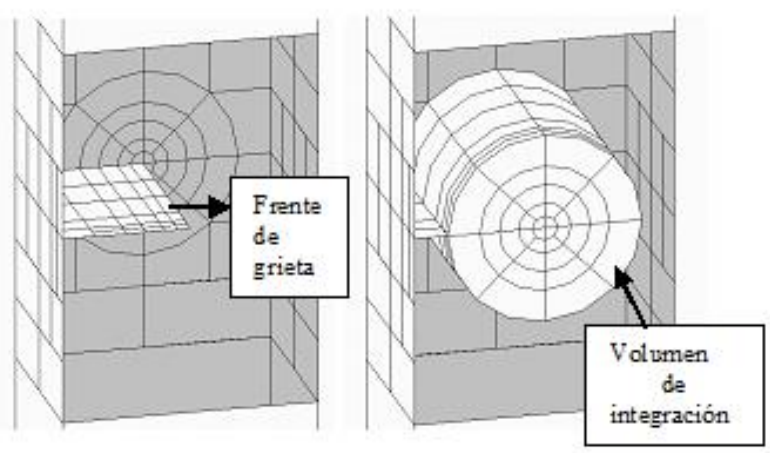

Figura 3. Discretización del contorno y dominios de integración para la evaluación de la integral J.

Fuente: elaboración propia.

La integral $J$ está relacionada con los factores de intensidad de esfuerzo si el material cumple con la teoría de la mecánica de fractura lineal-elástica (MFLE), a través de la siguiente expresión:

$$
J=\frac{1-v^{2}}{E}\left(K_{I}^{2}+K_{I I}^{2}\right)+\frac{1-v}{E} K_{I I I}^{2}
$$

donde $E$ es el módulo de elasticidad del material. Luego, a partir de un proceso de desacople de la ecuación (13), es posible obtener los factores de intensidad de esfuerzo de acuerdo con el procedimiento desarrollado por Balderrama [16].

\section{Cálculo de la velocidad de crecimiento de grieta por fatiga}

Una vez definidos los valores del factor de intensidad de esfuerzo en sus tres modos $K_{I}, K_{I I}$ y $K_{I I I}$ a través del programa computacional diseñado para tal fin, es posible determinar el rango equivalente de ese factor $\left(\Delta K_{e q}\right)$, a través de la siguiente ecuación propuesta por Gerstle [17]:

$$
\left(\Delta K_{e q}\right)^{2}=\left(\Delta K_{I}+B\left|\Delta K_{I I I}\right|\right)^{2}+2\left(\Delta K_{I I}\right)^{2}
$$

donde el intervalo del factor de intensidad de esfuerzo asociado a un esfuerzo máximo y a un esfuerzo mínimo se define como 


$$
\Delta K_{i}=K \max _{i}-K \min _{i}, i=I, I I, I I I
$$

La constante $B$ es un factor determinado empíricamente; en este trabajo se utilizó $B=1$. Este valor arroja resultados satisfactorios en la simulación de crecimiento de grietas por fatiga en tres dimensiones, según el trabajo realizado por Gerstle [17].

Con el fin de simular el crecimiento de la grieta, se estableció que el tamaño de cada incremento de extensión de la grieta $(\Delta a)$ fuese de $(0,1) a_{i}$, siendo $a_{i}$ la longitud inicial de la grieta; los resultados que se presentan en este trabajo corresponden a 7 incrementos de extensión de grieta. En cada incremento fue necesario remallar nuevamente las superficies de grieta.

La evaluación computacional de la velocidad de crecimiento de grieta por fatiga se obtuvo luego de calcular el rango del factor de intensidad de esfuerzo equivalente $\Delta K_{e q}$, a través de la ecuación (14) y con base en unas condiciones de temperatura y frecuencia de carga específicas. Para el cálculo de esa velocidad se utilizó el modelo de Kim y Wang [5], descrito a continuación:

$$
\frac{d a}{d N}=f^{-n m} A \exp \left[-\frac{\Delta H_{t h}-\gamma \log \Delta K_{e q}}{R T}\right]
$$

donde $d a / d N$ es la velocidad de crecimiento de grieta, $f$ es la frecuencia de la carga, $\Delta H_{t h}$ es la energía de activación aparente (independiente de $\Delta K$ ), $\Delta K$ es el rango de intensidad de esfuerzo, $R$ la constante universal de los gases $(=8,31 \mathrm{~J} / \mathrm{mol} \mathrm{K})$ y $T$ la temperatura absoluta.

Los resultados obtenidos con este modelo fueron comparados con los valores ajustados al modelo de crecimiento de grieta tradicional, presentado por Paris y Erdogan [18], y descrito por la siguiente expresión:

$$
\frac{d a}{d N}=C\left(\Delta K_{e q}\right)^{m}
$$

Los valores de la velocidad de crecimiento de grieta ajustados por el modelo de Paris y Erdogan corresponden a resultados experimentales del proceso de fatiga del CPVC a diferentes temperaturas $\left(23,50\right.$ y $\left.70^{\circ} \mathrm{C}\right)$ y frecuencias de carga $(0,1 ; 1$ y $10 \mathrm{~Hz})$.

\section{Resultados}

En esta sección se muestra la aplicación de la metodología computacional, para la determinación de los parámetros fractomecánicos involucrados en el análisis de crecimiento de grietas, a través del MDEC. Además, se evalúa el modelo matemático propuesto por Kim y Wang [5], para predecir la velocidad de crecimiento de una grieta por fatiga, cuando varían las condiciones de ensayo, específicamente, la temperatura y la frecuencia de la carga aplicada, en un ejemplo sencillo de grieta con frente recto.

Para cada caso se comparan los resultados con los obtenidos por otros autores, mediante métodos experimentales o soluciones analíticas ajustados al modelo tradicional de Paris y Erdogan [18].

Para el ejemplo, el material empleado es el CPVC, cuyas propiedades mecánicas son las siguientes: módulo de Young $E=2482,11 \mathrm{MPa}$, resistencia a la fluencia $\sigma_{y}=$ 53,78 MPa, módulo de Poisson $v=0,43$, coeficiente de expansión térmica $\alpha=3,4 \times 10^{-5} /{ }^{\circ} \mathrm{C}$ y conductividad térmica $\lambda=0,95 \mathrm{~W} /{ }^{\circ} \mathrm{C} . \mathrm{m}$. En ningún caso se considera la influencia de la temperatura en la variación de estos parámetros. Los parámetros $n m, \gamma, \Delta H_{t h}$ y $A$ del modelo de Kim y Wang (ecuación 16) fueron obtenidos para el CPVC por Saghir et al. [7]; estos valores son los siguientes: $\mathrm{nm}=0,2027, \gamma=12,7, \Delta H_{t h}=33300 \mathrm{~J} / \mathrm{mol}$ y $A=6,976$.

Los parámetros $C$ y $m$ del modelo de Paris y Erdogan (ecuación 17) se obtuvieron experimentalmente para el CPVC, a partir del ajuste de las curvas de fatiga ( $L o g$ $d a / d N$ vs. $\log \Delta K$ ) a temperaturas de 23,50 y $70{ }^{\circ} \mathrm{C}$ y frecuencias de carga 0,$1 ; 1$ y $10 \mathrm{~Hz}$. Estos resultados se presentan en el trabajo realizado por Saghir et al. [7], los cuales se muestran en la tabla 1.

Tabla 1. Constantes $m$ y $C$ del modelo de Paris para el $\mathrm{CPVC}$ a diferentes frecuencias y temperaturas.

\begin{tabular}{cccc}
\hline $\begin{array}{c}\text { Temperatura } \\
\left({ }^{\circ} \mathbf{C}\right)\end{array}$ & $\begin{array}{c}\text { Frecuencia } \\
(\mathbf{H z})\end{array}$ & $\begin{array}{c}\text { Exponente } \\
\boldsymbol{m}\end{array}$ & $\begin{array}{c}\text { Parámetro } \\
\boldsymbol{C}\end{array}$ \\
\hline 23 & 0,1 & 2 & $1,00 \times 10^{-6}$ \\
& 1 & 2,3979 & $9,00 \times 10^{-7}$ \\
& 10 & 2,292 & $8,00 \times 10^{-7}$ \\
50 & 0,1 & 2,5702 & $6,00 \times 10^{-6}$ \\
& 1 & 2,465 & $2,00 \times 10^{-6}$ \\
& 10 & 2,2665 & $1,65 \times 10^{-6}$ \\
70 & 0,1 & 2,8074 & $1,00 \times 10^{-5}$ \\
& 1 & 2,4022 & $5,00 \times 10^{-6}$ \\
& 10 & 2,0205 & $3,82 \times 10^{-6}$ \\
\hline
\end{tabular}




\subsection{Probeta con grieta lateral}

Este primer ejemplo corresponde a una probeta rectangular que se modela en condiciones de esfuerzo plano, cuya longitud de grieta inicial es $a_{i}=10 \mathrm{~mm}$, el ancho de la probeta es $W=20 \mathrm{~mm}$, la altura $h=120 \mathrm{~mm}$ y el espesor $t=15 \mathrm{~mm}$. La grieta se encuentra en $h=60$ $\mathrm{mm}$. El modelo discretizado se muestra en la figura $4 \mathrm{y}$ está compuesto inicialmente por 1.127 nodos y 228 elementos de contorno, de los cuales 560 nodos y 70 elementos de contorno pertenecen a las superficies de la grieta. Para el cálculo de $J$ se construyeron 4 anillos de celdas con radios que varían entre 20 y $75 \%$ de la longitud de la grieta, compuestos por 216 celdas y 1.278 nodos de celda.

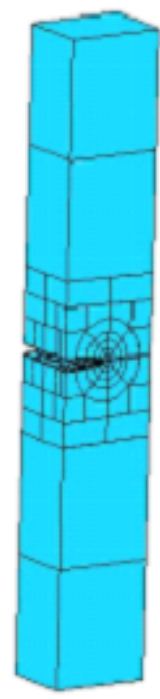

$23^{\circ} \mathrm{C}$

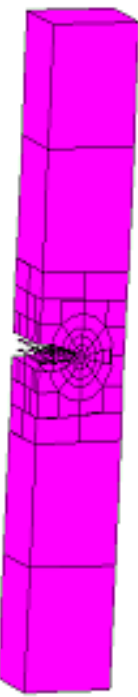

$50^{\circ} \mathrm{C}$

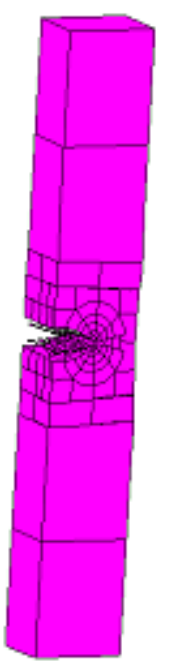

$70^{\circ} \mathrm{C}$
Figura 4. Apertura de grieta por fatiga a diferentes temperaturas.

El proceso de simulación de crecimiento de grieta fue generado por un ciclo de carga de amplitud constante con una relación $R\left(=\sigma_{\min } / \sigma_{\max }\right)$ de 0,20 y un rango de esfuerzo $\Delta \sigma\left(=\sigma_{\text {máx }}-\sigma_{\text {mín }}\right)$ de $13,3 M P a$. Las temperaturas $\theta$ del medio isotérmico son 23,50 y $70{ }^{\circ} \mathrm{C}$ y las frecuencias de carga son $0,1,1$ y $10 \mathrm{~Hz}$. La probeta se somete a fatiga por tracción uniforme en la dirección perpendicular a la grieta.

Los factores de intensidad de esfuerzo $K_{I}, K_{I I}$ y $K_{I I I}$, obtenidos a través del método dual de los elementos de contorno, se normalizaron utilizando la siguiente expresión:

$$
K_{i}^{*}=K_{i} / \alpha E \theta \sqrt{W} ; i=I, I I, I I I
$$

donde $\alpha, E, \theta$ y $W$ son el coeficiente de expansión térmica, el módulo de elasticidad, la temperatura y el ancho de la probeta, respectivamente.

\subsubsection{Efecto de la temperatura de ensayo sobre la velocidad de crecimiento de grieta $(\mathrm{da} / \mathrm{dN})$}

Para esta primera geometría, el efecto de la temperatura del medioambiente sobre la velocidad de extensión de la grieta durante el proceso de fatiga del CPVC se puede observar claramente en la figura 5, donde se grafican los datos de $d a / d N$ vs. $\Delta K$ en escala logarítmica a diferentes temperaturas ajustados por el modelo tradicional de Paris y Erdogan para una frecuencia de carga fija de $1 \mathrm{~Hz}$, donde los valores de $\Delta K$ y $d a / d N$ corresponden a los obtenidos computacionalmente.

En esta gráfica se observa que la velocidad de crecimiento de grieta aumenta con la temperatura a un valor fijo de $\Delta K$, es decir, que la resistencia del material al crecimiento de grieta disminuye al aumentar la temperatura del medio, lo cual concuerda con los resultados obtenidos en los trabajos realizados por Kim y Mai [2], Irfan-ul-Haq y Merah [3] y Parson et al. [6].

Desde el punto de vista de la microestructura del material, el hecho de que la velocidad de crecimiento de grieta por fatiga aumente con la temperatura del medioambiente se debe al fácil desplazamiento y al desenredo de las cadenas poliméricas, como resultado de una disminución de la fuerza de enlace y un incremento en la actividad molecular a altas temperaturas.

Con el fin de validar los resultados de la simulación computacional, obtenidos para este primer ejemplo, empleando el modelo de Kim y Wang [5], bajo ciertas condiciones de temperatura y frecuencia de carga fija, en la figura 6 se comparan esos resultados con valores de referencia provenientes del trabajo experimental. Estos valores se ajustan al modelo tradicional de Paris (modelo de referencia). Los resultados expresados en la gráfica corresponden al logaritmo de la velocidad de crecimiento de grieta promedio obtenido para cada temperatura a una frecuencia fija de $1 \mathrm{~Hz}$. 


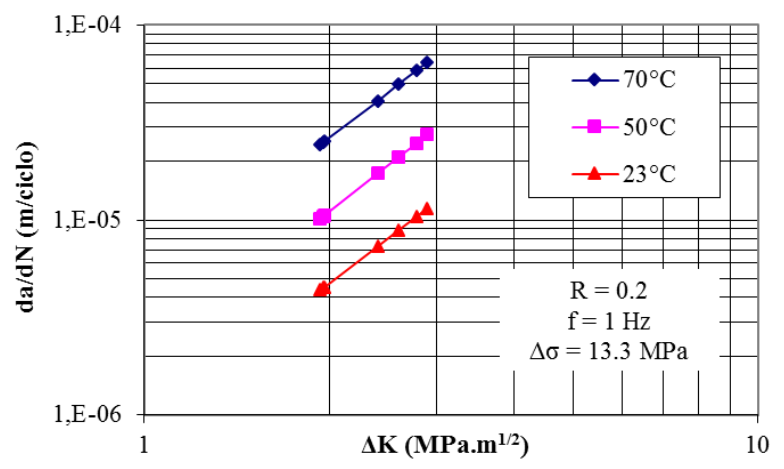

Figura 5. Velocidades de crecimiento de grieta por fatiga a diferentes temperaturas para el CPVC a la frecuencia de carga de $1 \mathrm{~Hz}$.

Como se puede ver en la figura, el modelo propuesto por $\mathrm{Kim}$, en este caso, genera valores aproximados a los valores de referencia. A una temperatura de $23{ }^{\circ} \mathrm{C}$ se logra una mejor aproximación a los valores reales, ya que el error relativo promedio es de $1,09 \%$, mientras que a temperaturas de 50 y $70{ }^{\circ} \mathrm{C}$, los errores relativos promedios son 3,67 y $2,62 \%$, respectivamente.

La malla deformada, como resultado de la simulación en cada caso, se puede observar en la figura 4. Allí se observa que la deformación de la probeta y la apertura de grieta son más pronunciadas en el caso de fatiga a $70^{\circ} \mathrm{C}$.

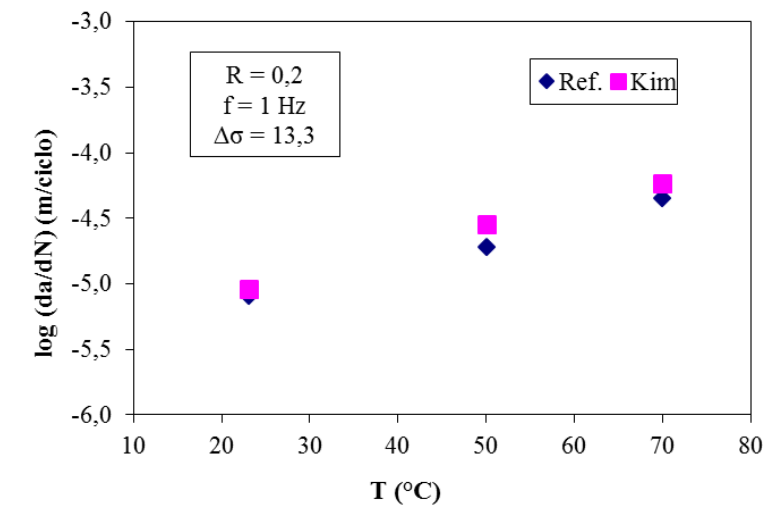

Figura 6. Velocidad de crecimiento de grieta $(\mathrm{d} a / \mathrm{dN})$ promedio vs. temperatura.

\subsubsection{Efecto de la frecuencia de carga sobre la velocidad de crecimiento de grieta $(\mathrm{da} / \mathrm{dN})$}

Para ilustrar este efecto, se tomaron los datos de velocidad de crecimiento de grieta, obtenidos mediante el modelo de Paris y los valores de $\Delta K$ computacional. En la figura 7 se grafican los datos de $d a / d N$ vs. $\Delta K$ en escala logarítmica a diferentes frecuencias para una temperatura fija de $50{ }^{\circ} \mathrm{C}$ y se aprecia claramente que, al aumentar la frecuencia de la carga durante el proceso de fatiga a una temperatura fija, la velocidad de crecimiento de grieta disminuye, lo cual concuerda con lo reportado por Merah et al. [19].

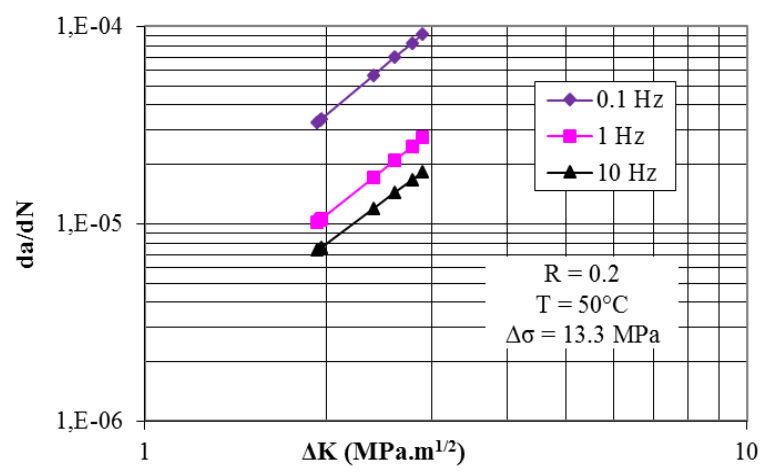

Figura 7. VCG $(\mathrm{d} a / \mathrm{dN})$ vs. $\Delta \mathrm{K}$ a diferentes frecuencias de carga para el CPVC a $50{ }^{\circ} \mathrm{C}$.

Con la finalidad de validar los resultados de la simulación computacional, mediante el modelo de Kim y Wang, para evaluar el efecto del cambio de frecuencia de carga sobre la velocidad de crecimiento de grieta en la fatiga del CPVC a una temperatura fija, se muestra en la figura 8 una comparación entre los valores computacionales del logaritmo de la velocidad de crecimiento de grieta promedio obtenidos con el modelo de Kim y los valores de referencia ajustados al modelo de Paris.

Estos resultados reflejan que los valores de la velocidad de crecimiento de grieta calculados con el modelo de Kim y Wang se aproximan más a los valores reales ajustados al modelo de Paris, cuando la frecuencia de carga es de $10 \mathrm{~Hz}$, ya que el error relativo promedio es de $2,73 \%$ mientras que a 0,1 y $1 \mathrm{~Hz}$ es de $-3,44$ y 3,67 $\%$, respectivamente.

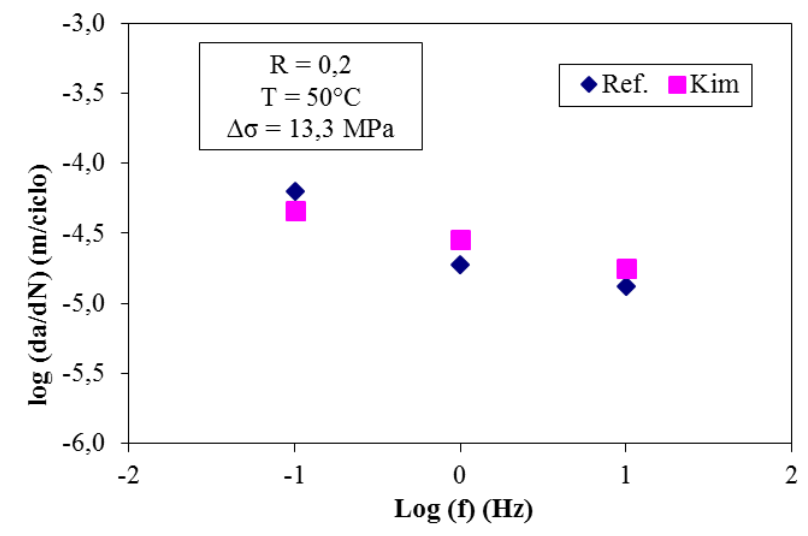

Figura 8. VCG (da/dN) promedio vs. frecuencia de carga. 


\section{Conclusiones}

Se logró modelar el crecimiento de grieta a través de un algoritmo que permitió, en primer lugar, la determinación de los parámetros fractomecánicos (deformaciones, esfuerzos $y$ factor de intensidad de esfuerzo), implementando el método dual de los elementos de contorno (MDEC). Estos parámetros fueron el punto de partida para la aplicación del modelo matemático de crecimiento de grieta propuesto por los investigadores Kim y Wang, que incluye la temperatura y la frecuencia de carga como variables independientes que afectan directamente las velocidades de crecimiento de grieta.

En este sentido, la herramienta computacional que utiliza el MDEC jugó un papel fundamental, ya que permitió determinar los factores de intensidad de esfuerzo para cada uno de los casos estudiados, y dio un aporte al trabajo netamente experimental realizado por Saghir et al. [7].

La velocidad de crecimiento de grieta por fatiga se ve influenciada por las condiciones de ensayo: temperatura y frecuencia de carga. La resistencia al crecimiento de grieta por fatiga incrementa con el aumento de la frecuencia y disminuye con el aumento de la temperatura.

El análisis comparativo realizado entre los resultados obtenidos aplicando el modelo de crecimiento de grieta propuesto por Kim y Wang y los resultados conocidos aplicando el modelo de Paris y Erdogan permite concluir que el modelo de crecimiento propuesto por Kim y Wang funciona bastante bien para predecir las velocidades de extensión de grieta en ensayos de fatiga del CPVC, sobre todo a altas frecuencias (10 Hz en este caso) y a una determinada temperatura $\left(23,50\right.$ o $70{ }^{\circ} \mathrm{C}$, en este caso).

\section{Referencias}

[[1] A. F. Liu, Mechanics and Mechanisms of Fracture: An Introduction. ASM International, 2005.

[2] H.-S. Kim y Y.-W. Mai, "Effect of temperature on fatigue crack growth in unplasticized polyvinyl chloride," J. Mater. Sci., vol. 28, no. 20, pp. 5479 -5485, 1993. doi: 10.1007/BF00367818

[3] M. Irfan-ul-Haq y N. Merah, "Effect of Temperature on Fatigue Crack Growth in CPVC," J. Press. Vessel Technol., vol. 125, no. 1, pp. 71-77, 2003. doi: $10.1115 / 1.1523070$

[4] R. W. Hertzberg, J. A. Manson, y M. Skibo, "Frequency sensitivity of fatigue processes in polymeric solids," Polym. Eng. Sci., vol. 15, no. 4, pp. 252-260, 1975. doi: 10.1002/pen.760150404

[5] H. S. Kim y X. M. Wang, "Temperature and frequency effects on fatigue crack growth of uPVC," $J$. Mater. Sci., vol. 29, no. 12, pp. 3209-3214, 1994. doi: 10.1007/BF00356664

[6] Parson M, Stepanov EV, Hiltner A, Baer E., "Fatigue and Creep Crack Propagation Kinetics in PVC Pipe Material," in The Annual Technical Conference (ANTEC) of the Society of Plastics Engineers, 2002, pp. 426-430.

[7] F. Saghir, N. Merah, Z. Khan, y A. Bazoune, "Modeling the combined effects of temperature and frequency on fatigue crack growth of chlorinated polyvinyl chloride (CPVC)," J. Mater. Process. Technol., vol. 164-165, pp. 1550-1553, 2005. doi: 10.1016/j.jmatprotec.2005.02.206

[8] M.H. Aliabadi, The Boundary Element Method, Applications in Solids and Structures Vol. 2. John Wiley \& Sons, 2002.

[9] J. Mackerle, "FEM and BEM in the context of information retrieval," Comput. Struct., vol. 80, no. 2021 , pp. 1595-1604, 2002. doi: 10.1016/S00457949(02)00114-1

[10] T. A. Cruse, "Numerical solutions in three dimensional elastostatics," Int. J. Solids Struct., vol. 5, no. 12 , pp. $1259-1274$, 1969. doi: 10.1016/00207683(69)90071-7

[11] T. A. Cruse, "Numerical Evaluation of Elastic Stress Intensity Factors by the Boundary Integral Equation Method, The Surface Crack: Physical Properties and Computational Solutions," J.L. Swedlow (Ed.), ASME, New York, 1972, pp. 153-170

[12] T. A. Cruse, "BIE fracture mechanics analysis: 25 years of developments," Comput. Mech., vol. 18, no. 1, pp. 1-11, 1996. doi: 10.1007/BF00384172

[13] A. Portela, M. H. Aliabadi, y D. P. Rooke, "The dual boundary element method: Effective implementation for crack problems," Int. J. Numer. Methods Eng., vol. 33, no. 6, pp. 1269-1287, 1992. doi: $10.1002 /$ nme. 1620330611

[14] Y. Mi y M. H. Aliabadi, "Dual boundary element method for three-dimensional fracture mechanics analysis," Eng. Anal. Bound. Elem., vol. 10, no. 2, pp. 161-171, 1992. doi: 10.1016/0955-7997(92)90047-B 
[15] D. N. Dell'Erba, Thermoelastic fracture mechanics using boundary elements. WIT Press, 2002.

[16] R. Balderrama, "Implementación de la Integral J de Dominio para el Análisis Tridimensional de Grietas en Problemas Termoelásticos utilizando el Método de Elementos de Contorno", trabajo de fin de maestría, Universidad Central de Venezuela, Caracas, Venezuela, 2004.

[17] W. H. Gerstle, "Finite and Boundary Element Modelling of Crack Propagation in Two and Three Dimensions using Interactive Computer Graphics", tesis doctoral, Cornell University, New York, 1985.

[18] P.C. Paris, F. Erdogan, "A Critical Analysis of Crack Propagation Laws," J. Basic Eng., vol. 85, no. 4, pp. 528533, 1963. doi: 10.1115/1.3656900

[19] N. Merah, F. Saghir, Z. Khan, y A. Bazoune, “A study of frequency and temperature effects on fatigue crack growth resistance of CPVC," Eng. Fract. Mech., vol. 72, no. 11, pp. 1691-1701, 2005. doi: 10.1016/j.engfracmech.2004.12.002 